() Turkish Society of Radiology 2019

\section{Undifferentiated pancreatic carcinoma with osteoclast-like giant cells}

Lan Zhang

From the Department of MRI (L.Z. $ه 13837187787 @ 163 . c o m)$, the First Affiliated Hospital of Henan University of Chinese Medicine, Zhengzhou, China.

\section{Dear Editor,}

I read with great interest pictorial essay entitled "Pancreas ductal adenocarcinoma with cystic features on cross-sectional imaging: radiologic-pathologic correlation" by Seo Yeon Youn et al. (1) in the February 2018 issue of Diagnostic and Interventional Radiology. The author described the radiologic features of pancreatic ductal adenocarcinomas (PDAC) with cystic appearance. We would like to share a very rare subtype of PDAC, undifferentiated pancreatic carcinoma with osteoclast-like giant cells (UPC-OGC) presenting as a cystic mass.

A 57-year-old male was admitted with epigastric pain and weight loss for one month. Most indices of laboratory tests were normal except for slight anemia. Abdominal computed tomography (CT) and magnetic resonance imaging (MRI) revealed an approximately $12 \mathrm{~cm} \times 9 \mathrm{~cm} \times 8 \mathrm{~cm}$, round-like, well-defined and cystic mass with inhomogeneous enhancement in the left upper abdomen (Fig. a-d). The tumor appeared to originate from the pancreatic tail with invasion to the spleen, abutting parts of the stomach and colon. The patient underwent complete resection. Grossly, a $12 \mathrm{~cm}$ $\times 9 \mathrm{~cm} \times 9 \mathrm{~cm}$ multilocular mass was located in the tail of pancreas with adhesion to the surrounding tissue. There were cysts filled with hemorrhagic and necrotic contents on the cut surface. Microscopically, the tumor was found to have pleomorphic mononuclear cells with multinucleated OGC (Fig. e). Immunohistology revealed that OGCs were positive for $C D$ 68, which is a characteristic histiocytic marker of OGC (Fig. f). However, follow-up CT discovered a huge and recurrent mass in the left abdomen, surrounding the left kidney with partial invasion to the kidney 6 months after the operation.

UPC-OGC is a rare and peculiar pancreatic tumor known to originate from pancreatic ductal epithelium and categorized as a variant subtype of PDAC (2). Most UPC-OGCs have an insidious onset and are found as large tumors upon detection with a tendency towards early recurrence, even after surgical removal. Since the tumor progresses rapidly, UPC-OGC often appears as a heterogeneous cystic mass with hemorrhage and necrosis. CT and MRI demonstrated a well-demarcated, cystic tumor with obvious enhancement of septations and capsule within the tumor in our case. Hence, an inhomogeneous mass with cystic structures and nonuniform enhancement is a typical radiologic finding of UPCOGC. However, the definite diagnosis of UPC-OGC could be obtained through histopathology and immunohistochemistry. The presence of OGC is the histologically distinguishing feature of UPC-OGC (3). OGC lacks morphologic atypia and typically stains for histomonocytic markers (CD68).

It has been reported that small-sized UPC-OGC without invasion to the surrounding tissues may have a more favorable prognosis after complete excision (4). Therefore, an early diagnosis is the key
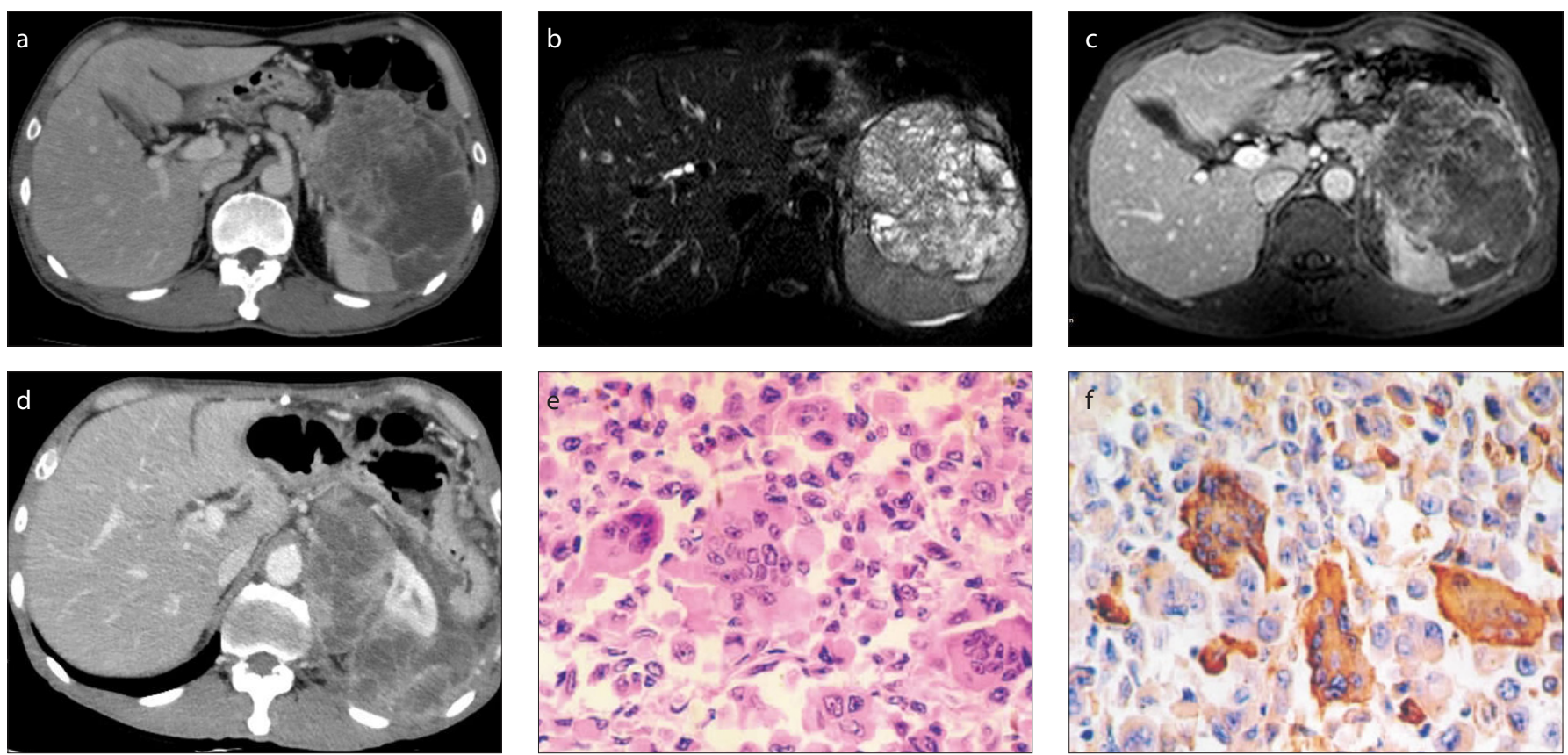

Figure. a-f. undifferentiated pancreatic carcinoma with osteoclast-like giant cells in a 57-year-old male with epigastric pain. Contrast-enhanced CT (a) demonstrates a large cystic and solid mass with inhomogeneous enhancement. Axial T2-weighted imaging (b) shows a large miscellaneous intensity tumor. Contrast-enhanced MRI (c) demonstrates a heterogeneous mass with inhomogeneous enhancement of septations and capsule within the tumor. Contrast-enhanced CT (d) demonstrates a huge, multifocal, recurrent intraperitoneal mass with invasion to the residual kidney in the left abdomen. Hematoxylin and eosin staining $(\times 20)($ e) shows the tumor is composed of pleomorphic mononuclear cells and osteoclast-like giant cells. Immunohistochemical staining $(\times 40)(f)$ shows CD68 expression in osteoclast-like giant cells. 
concern for UPC-OGC. Fine needle aspiration is recommended as an effective modality to achieve a precise diagnosis. In our case, although the tumor was huge and invaded abutting organs, complete resection of tumor and involved organs was still the optimal treatment for this patient. However, the patient was found to have recurrence at nearly the same location within 6 months. Since the recurrent tumor was more invasive than the original one, the patient lost his second chance of surgery.

\section{Conflict of interest disclosure}

The author declared no conflicts of interest.

\section{References}

1. Youn SY, Rha SE, Jung ES, Lee IS. Pancreas ductal adenocarcinoma with cystic features on cross-sectional imaging: radiologic-pathologic correlation. Diagn Interv Radiol 2018; 24:5-11. [CrossRef]

2 Bosman FT, Carneiro F, Hruban RH, Theise ND. WHO Classification of Tumors of the Digestive System. 4th ed. Lyon: International Agency for Research on Cancer (IARC) 2010:281-295.

3 Reid MD, Muraki T, HooKim K, et al. Cytologic Features and clinical implications of undifferentiated carcinoma with osteoclastic giant cells of the pancreas: An analysis of 15 cases. Cancer Cytopathol 2017; 125:563-575. [CrossRef]

4 Muraki T, Reid MD, Basturk O, et al. Undifferentiated carcinoma with osteoclastic giant cells of the pancreas: clinicopathologic analysis of 38 cases highlights a more protracted clinical course than currently appreciated. Am J Surg Pathol 2016;40:1203-1216. [CrossRef]

Received 27 May 2018; revision requested 28 May 2018; last revision received 9 July 2018; accepted 17 July 2018.
Published online 8 January 2019.

DOI 10.5152/dir.2019.18261

You may cite this article as: Zhang L. Undifferentiated pancreatic carcinoma with osteoclast-like giant cells. Diagn Interv Radiol 2019; 25:173-174.

\section{Author's Reply}

\section{Sung Eun Rha}

From the Department of Radiology, Seoul St. Mary's Hospital, The Catholic University of Korea, College of Medicine, Seoul, South Korea.

We thank the author for his interest in our article, "Pancreas ductal adenocarcinoma with cystic features on cross-sectional imaging: radiologic-pathologic correlation" (1), and for sharing the rare case showing cystic features on CT and MRI. Our paper focused on the classification of pancreas ductal adenocarcinoma with cystic features based on histopathologic types. Therefore, we did not cover every histologic subtype of pancreatic ductal adenocarcinoma.

As the author commented, undifferentiated pancreatic carcinoma with osteoclast-like giant cells (UPC-OGC) is a very rare histologic subtype of pancreatic ductal adenocarcinoma, comprising less than $1 \%$ of all exocrine pancreatic tumors (2). Less than 50 cases of UPC-OGC have been reported in the English literature and imaging findings of UPC-OGC have been rarely reported (3). More than $80 \%$ of the report- ed cases present as a pancreatic mass larger than $5 \mathrm{~cm}$ and several authors reported UPC-OGC seen as a large, predominantly cystic neoplasm with various extents of hemorrhage and necrosis, which are similar to the author's case (4). According to our histopathologic classifications of cystic changes associated with pancreatic ductal adenocarcinoma, the cystic feature of UPC-OGC may correspond to degenerative cystic changes formed by tumor necrosis containing necrotic and hemorrhagic tissue in the cavity. Due to rarity of these tumors, imaging findings cannot be characterized, but this rare subtype of pancreatic adenocarcinoma can show extensive cystic degeneration.

We appreciate the author's interest in our article.

\section{References}

1. Youn SY, Rha SE, Jung ES, Lee IS. Pancreas ductal adenocarcinoma with cystic features on cross-sectional imaging: radiologic-pathologic correlation. Diagn Interv Radiol 2018; 24:5-11. [CrossRef]

2. Bauditz J, Rudolph B, Wermke W. Osteoclast-like giant cell tumors of the pancreas and liver. World J Gastroenterol 2006; 12:78787883. [CrossRef]

3. Yang KY, Choi Jl, Choi MH, et al. Magnetic resonance imaging findings of undifferentiated carcinoma with osteoclast-like giant cells of pancreas. Clin Imaging 2016; 40:148-151. [CrossRef]

4. Hur YH, Kim HH, Seoung JS, et al. Undifferentiated carcinoma of the pancreas with osteoclast-like giant cells. J Korean Surg Soc 2011; 81:146-150. [CrossRef] 